\title{
Investigación en química de los departamentos de la Universidad de Castilla La Mancha
}

\author{
Chemistry research at Castilla-La Mancha University
}

\author{
María Luisa Pérez Aliende \\ UCLM. Biblioteca General de Cuenca, Avda.de los Alfares 42, Cuenca, mluisa.perez@uclm.es.
}

\begin{abstract}
Resumen
En este estudio bibliométrico se pretende dar a conocer la producción y el impacto de la actividad científica de la Universidad de Castilla-La Mancha en el campo de la química durante el período 2000-2005. Para ello se han estudiado los trabajos publicados en revistas indizadas en las bases de datos Science Citation Index y Journal Citation Reports, especialmente adecuadas para su utilización en los estudios bibliométricos por indizar las revistas más representativas de la actividad científica internacional. Los resultados permitirán conocer y comparar la evolución de la producción de los departamentos, así como estudiar aspectos relacionados con la colaboración científica, entre autores y también entre instituciones, tanto a nivel nacional como internacional. Para conocer la visibilidad de la investigación se recurrirá al factor de impacto de las revistas en que publican los investigadores de estos departamentos, lo que permitirá conocer en cierto modo la calidad de las revistas según su interés científico
\end{abstract}

Palabras clave: Actividad científica. Química. Visibilidad de la investigación. Revistas científicas. Colaboración científica.

\section{Introducción}

La ciencia y la tecnología han adquirido gran importancia en la sociedad actual, por la gran influencia que ejercen en todos los aspectos de nuestra vida. Este hecho hace necesario conocer el rendimiento de la actividad científica pues son muchos los recursos que se le han destinado, y para ello es necesario realizar una evaluación (Sancho, 1990). Entre los indicadores con los que hay que trabajar para su evaluación hay que destacar los que permiten medir el output, es decir, los resultados de la ciencia, producto final de toda investigación (Prat, 2001), que se transmiten en forma de publicaciones científicas como son los artículos de revista, actas de congresos o patentes

La generación de nuevo conocimiento es un factor decisivo para realizar la evaluación de una institución que se dedica a generar ciencia (Arencibia et al., 2007). Y la universidad además

\begin{abstract}
This paper presents a bibliometric study which tries to find out the output and impact of the research activities in the field of Chemistry at the University of Castilla-La Mancha during the period 2000-2005. The data used in this study were obtained from the Science Citation Index and Journal Citation Reports databases, specially adapted to be used in bibliométric studies, by indexing the most important international journals. Results report data on productivity output of the departments, and show the scientific collaboration patterns, between authors, via the coauthorship index and the degree of domestic and international collaboration. The research visibility will be known measuring the impact factor and quartiles as a tool for ranking and categorizing journals in which it was published.
\end{abstract}

Keywords: Scientific activity. Chemistry. Research visibility. Scientific journals. Scientific collaboration.

de destacar por su labor docente también lo hace por su actividad investigadora. No en vano el sistema universitario español representaba en los primeros años de esta década el $66 \%$ de la producción española con visibilidad internacional (Grupo Scimago, 2007). Este porcentaje es similar a los valores que alcanza la Universidad de Castilla-La Mancha (UCLM) en la región manchega, donde el entorno científico está básicamente integrado por el personal de la misma. Esta comunidad se encuentra en estos momentos en pleno desarrollo tecnológico y son muchos los esfuerzos y políticas regionales que van destinados a ello. En este sentido dos sectores se vieron especialmente favorecidos por las ayudas a la investigación recibidas, el agroalimentario y la industria química, sector más importante de los considerados de alta tecnología, pues no hay que olvidar la importancia de la industria petroquímica en la región. 


\section{Objetivos}

El objetivo que se pretende con este estudio es conocer la actividad y visibilidad internacional de los trabajos realizados por los departamentos del área de la química en la Universidad de Castilla-La Mancha. Para ello se estudian aspectos relacionados con la producción, la calidad y la repercusión de la ciencia, en particular: la producción de los departamentos; la colaboración científica, tanto a nivel de autores como de instituciones; y la visibilidad internacional de los resultados de las investigaciones.

\section{Metodología}

Se analiza la producción científica en química de los cuatro departamentos de la UCLM que abordan esta materia: Química Inorgánica, Orgánica y Bioquímica (QIOB), Química Analítica y Tecnología de los Alimentos (QATA), Química Física (QF) e Ingeniería Química (IQ). No se ha tenido en cuenta la actividad del Instituto de Tecnología Química y Medioambiental de Ciudad Real (ITQUIMA) por ser de reciente creación.

Para su realización se utilizaron los registros recuperados de la base de datos del ISI, Science Citation Index (SCl), accesible a través de la plataforma Web of Knowledge durante los años 2000 a 2005. Se utilizó también el Journal Citation Reports (JCR) por aportar los datos que nos permiten determinar de forma objetiva la importancia de una revista en su categoría temática y el factor de impacto.

La búsqueda realizada a partir de la afiliación de los autores, permitió recuperar todos los artículos indizados durante ese período en el SCI. Los registros recuperados se transfirieron a una base de datos en Procite 5.0, que permitió recuperar los ítems para su tratamiento estadístico. Se utilizó el sistema de asignación completa o recuento total, de modo que los sumatorios de los recuentos serán superiores al total real de los documentos (Gómez et al., 2003).

Los indicadores de actividad que se utilizan son: la producción científica por departamentos, la colaboración científica entre autores estudiando el índice de coautoría, y tasa de documentos coautorados; y entre instituciones a nivel interdepartamental, nacional e internacional. El factor de impacto de las revistas de publicación fue usado como indicador de visibilidad, organizándolas en cuartiles. Se utilizaron los datos correspondientes a 2005 del JCR.

\section{Resultados}

Durante los seis años objeto de estudio el personal de los departamentos ha publicado 673 documentos, es decir, el $36 \%$ del total publicado por la UCLM (1867) y recogido en el SCI durante ese período.

\subsection{Producción de los departamentos}

La evolución anual de la producción de los cuatro departamentos relacionados con la química queda plasmada en la siguiente tabla.

\begin{tabular}{ccccccc}
\hline & QIOB & QATA & QF & IQ & Total & $\%$ \\
\hline 2000 & 34 & 21 & 24 & 20 & 99 & 14,71 \\
2001 & 35 & 18 & 19 & 17 & 89 & 13,22 \\
2002 & 41 & 31 & 23 & 21 & 116 & 17,24 \\
2003 & 35 & 35 & 26 & 19 & 115 & 17,09 \\
2004 & 37 & 31 & 27 & 25 & 120 & 17,83 \\
2005 & 31 & 37 & 35 & 31 & 134 & 19,91 \\
\hline Total & 213 & 173 & 154 & 133 & 673 & \\
\hline$\%$ & 31,65 & 25,71 & 22,88 & 19,76 & & \\
\hline
\end{tabular}

Tabla I. Evolución anual de la productividad

El departamento de Química Inorgánica, Orgánica y Bioquímica (QIOB) es el más productivo de la Universidad en los últimos 6 años, responsabilizándose del $31,65 \%$ de los trabajos publicados por el área y del $11,41 \%$ de la UCLM. Química Analítica y Tecnología de los Alimentos (QATA) es el segundo departamento más activo con 173 trabajos. Química Física (QF) e Ingeniería Química (IQ) se responsabilizan del $42,6 \%$ de los trabajos restantes en esta materia. Si bien QIOB es el departamento con una actividad más homogénea durante este período cabe destacar el incremento de la actividad de los otros tres departamentos, destacando QATA con un $176 \%$.

A partir del año 2001 se observa un incremento constante de la actividad de estos departamentos, destacando el año 2005 con una subida porcentual del $2 \%$.

\subsection{La colaboración científica}

La colaboración científica se va a estudiar en dos apartados: por un lado, se analiza la colaboración entre autores a través del número de firmas por documento y la tasa de documentos coautorados; y, en un segundo, lugar la colaboración entre instituciones así como el tipo de colaboración -interdepartamental, nacional e internacional. 
4.2.1. La colaboración entre autores: índice de coautoría y tasa de documentos coautorados

En este apartado se estudia la distribución anual del número de autores por documento. La tabla siguiente muestra en la primera columna el numero de autores que han elaborado un documento, acompañado en las columnas sucesivas del número de documentos que han publicado esos autores cada año. En la última fila se expone el índice de coautoría o número medio de autores por trabajo para cada año objeto de estudio, que para este período es de 4,86 autores. Este valor alcanzo valores superiores a 5 en 2001 y 2002, para posteriormente decrecer y volver a estabilizarse entorno a 5 firmas en el 2005. El $98,4 \%$ de los trabajos se han realizado en colaboración con otros autores. Durante estos años el $43 \%$ de los artículos publicados han sido elaborados por $3(20,4 \%)$ ó $4(22,5 \%)$ autores. Sólo el $2 \%$ de los trabajos han sido elaborados por más de 10 autores.

\begin{tabular}{cccccccc}
\hline$N^{\circ}$ Aut. & 2000 & 2001 & 2002 & 2003 & 2004 & 2005 & Total \\
\hline 1 & 3 & 0 & 1 & 2 & 3 & 1 & 10 \\
2 & 8 & 3 & 11 & 13 & 3 & 6 & 44 \\
3 & 21 & 19 & 23 & 20 & 20 & 27 & 130 \\
4 & 24 & 15 & 16 & 23 & 33 & 32 & 143 \\
5 & 7 & 18 & 26 & 10 & 22 & 23 & 106 \\
6 & 15 & 8 & 11 & 11 & 11 & 13 & 69 \\
7 & 11 & 12 & 4 & 9 & 13 & 10 & 59 \\
8 & 3 & 6 & 6 & 9 & 9 & 9 & 42 \\
9 & 0 & 3 & 3 & 2 & 1 & 4 & 13 \\
10 & 0 & 0 & 4 & 2 & 0 & 1 & 7 \\
+10 & 3 & 2 & 2 & 3 & 0 & 3 & 13 \\
\hline Total & 95 & 86 & 107 & 104 & 115 & 129 & 636 \\
\hline I.Coaut. & 4,62 & 5,14 & 4,83 & 4,82 & 4,77 & 5 & 4,86 \\
\hline
\end{tabular}

Tabla II. Distribución y media anual de autores por documento

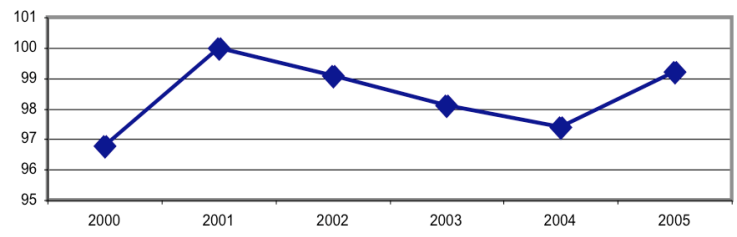

Figura 1. Evolución anual de la tasa de documentos coautorados

La tasa de documentos coautorados - definida como el porcentaje de trabajos con más de un firmante en relación con el total de documentos para un año determinado- se obtiene a partir de la tabla de distribución de autores por documento. Para este período es bastante alta, siendo del $100 \%$ en el año 2001 y manteniéndose en general durante todo el período en valores por encima del $97 \%$.

\subsubsection{Tipología de la colaboración}

A continuación se van a estudiar los documentos que han sido elaborados por dos o más instituciones siendo una de ellas, uno de los cuatro departamentos de la UCLM. Los trabajos pueden haber sido realizados sin colaboración, es decir, por autores pertenecientes al mismo o a varios de los departamentos de la Universidad; en colaboración nacional o en internacional a partir del trabajo conjunto con investigadores de otras instituciones españolas o extranjeras respectivamente.

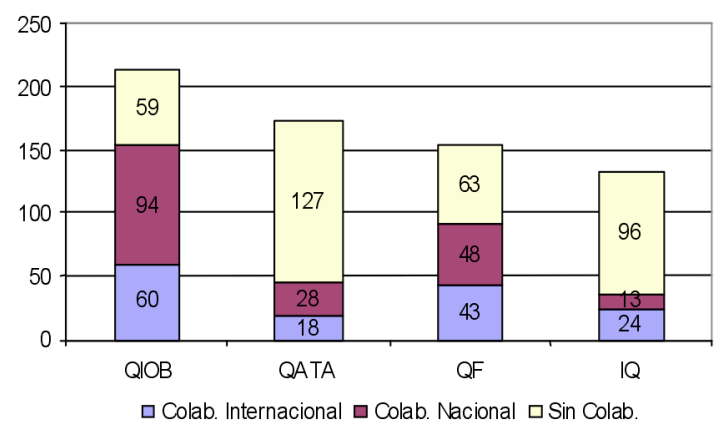

Figura 2. Distribución de la colaboración

Los departamentos que más publican sin ningún tipo de colaboración son QATA con 127 trabajos (73\%) e IQ con $96(72,2 \%)$, ambos mantienen una baja colaboración tanto nacional como internacional, con valores que oscilan entre el 10 y el $18 \%$. QIOB es el departamento con una mayor tasa de colaboración nacional —-94 trabajos $(44,1 \%)$ - con cifras respecto a la internacional y sin colaboración similares, 60 y 59 ítems respectivamente. QF destaca por realizar trabajos sin colaboración $(41 \%)$, pero colabora en el $59 \%$ de sus investigaciones con autores de otras instituciones nacionales e internacionales. Sumando la actividad de los cuatro departamentos, 345 trabajos $(51,3 \%)$ se han realizado sin colaboración, $183(27,2 \%)$ en colaboración nacional y $145(21,5)$ en internacional.

\subsubsection{La colaboración interdepartamental}

Se han realizado un total de 40 colaboraciones interdepartamentales en el total de 345 documentos publicados sin colaboración institucional, siendo QIOB el que más realiza (16), especialmente con el Centro Regional de Investigaciones Biomédicas (CRIB); seguido por IQ, que colabora en 6 ocasiones con el de Mecánica Aplicada e Ingeniería de Proyectos (MAIP). QATA es el que menos colabora con otros depar- 
tamentos y centros de investigación de la Universidad. Los departamentos del área de química colaboran fundamentalmente con el departamento MAIP y los centros de Investigación CRIB e IRICA (Instituto Regional de Investigación Científica Aplicada). Entre ellos existe un total de 10 colaboraciones, destacando el trabajo común de QIOB y QF en 3 ocasiones.

\subsubsection{La colaboración nacional}

Los cuatro departamentos del área de química han realizado un total de 183 documentos en colaboración. En la tabla IV se muestra la distribución de universidades españolas y centros de investigación del CSIC con los que se ha publicado un mínimo de 10 trabajos conjuntamente. Destaca la colaboración con la Universidad Rey Juan Carlos (31), la Universidad de Murcia (30) y la Universidad Complutense (20). Entre los centros del CSIC, destacan el Centro de Biología Molecular Severo Ochoa (18) o el Instituto de Química Médica (16). La colaboración con otros organismos, empresas u hospitales nacionales es bastante baja, razón por la que no se han incluido en la presente tabla.

\begin{tabular}{llc}
\hline \multicolumn{1}{c}{ Siglas } & \multicolumn{1}{c}{ Institución } & Docs. \\
\hline URJ & U.Rey Juan Carlos & 31 \\
UMU & U.Murcia & 30 \\
UCM & U. Complutense & 20 \\
CSIC-CBM & C. Biología Molecular & 18 \\
CSIC-IQM & Inst.Química Médica & 16 \\
UAB & U.Autónoma Barcelona & 13 \\
CSIC-IROC & Inst.Quím.Fís.Rocasolano & 12 \\
UNEX & U. Extremadura & 11 \\
CSIC-ICAT & Inst.Catal. Y Petroleoquímica & 11 \\
UAM & U. Autónoma Madrid & 10 \\
UAH & U. Alcalá de Henares & 10 \\
\hline
\end{tabular}

Tabla III. Colaboración con universidades españolas y centros de investigación

QIOB es el departamento con mayor colaboración nacional, realizándola en 94 documentos, y lo hace de una forma más habitual con 4 centros del CSIC: el Centro de Biología Molecular (18), el Instituto de Química Médica (16), el Instituto de Química Física Rocasolano (11) y el Instituto de Catálisis y Petroleoquímica (10). Tiene una actividad más frecuente con tres universidades madrileñas: la Universidad de Alcalá de Henares (9), la Universidad Autónoma (8) y la Universidad Complutense (8). Es destacable la colaboración de QF con la Universidad de Murcia (30), participando en el $19,5 \%$ de los trabajos publicados por este departamento.

\subsubsection{La colaboración internacional}

Un total de 145 documentos se han realizado en colaboración con instituciones de otros países. En la siguiente tabla aparecen los países con los que se trabajado en por lo menos 10 ocasiones. QIOB es el departamento que más colabora realiza con instituciones de otros países y lo hace fundamentalmente con centros de Francia (mayoritariamente con el CNRS) y de Estados Unidos. Sin embargo es QF el que mayor número de colaboraciones ha realizado con instituciones de un mismo país, en este caso Reino Unido (18), y en su mayoría con la Universidad de Bradford (12). Este país es a su vez con el que más colaboran los departamentos objeto de estudio de la UCLM, seguido de Francia, Italia, Estados Unidos, Alemania y Cuba.

\begin{tabular}{|c|c|c|c|c|c|}
\hline Países & Q & $\underset{\sigma}{\mathbb{\sigma}}$ & 岁 & $\stackrel{m}{o}$ & 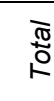 \\
\hline Reino Unido & 4 & 3 & 18 & 5 & 30 \\
\hline Francia & & 4 & 3 & 15 & 22 \\
\hline Italia & 2 & 2 & 4 & 9 & 17 \\
\hline EEUU & 1 & & 4 & 10 & 15 \\
\hline Alemania & 2 & & 9 & 1 & 12 \\
\hline Cuba & 6 & & & 4 & 10 \\
\hline
\end{tabular}

Tabla IV. Principales países con los que colaboran los departamentos

\subsection{Revistas de publicación y visibilidad de la investigación}

Se analiza la visibilidad de los departamentos a partir de las revistas que publican sus resultados de investigación. De este modo se ha recurrido al factor de impacto y a la asignación por cuartiles de las publicaciones que concentran el $50 \%$ de los trabajos.

\begin{tabular}{lcccc}
\hline \multicolumn{1}{c}{ Titulo de la revista } & $N^{\circ}$ & $\Sigma$ & $\%$ & $Q$ \\
\hline J.Organometallic Chemistry & 27 & 27 & 12,68 & 2 \\
Eur. J. Inorganic Chemistry & 19 & 46 & 21,60 & 2 \\
Organometallics & 16 & 62 & 29,11 & 1 \\
Dalton Transactions & 13 & 75 & 35,21 & 1 \\
J. Organic Chemistry & 8 & 83 & 38,97 & 1 \\
Synlett & 8 & 91 & 42,72 & 1 \\
Tetrahedron & 7 & 98 & 46,01 & 2 \\
Tetrahedron Letters & 6 & 104 & 48,83 & 2 \\
Inorganic Chemistry & 5 & 109 & 51,17 & 2 \\
\hline
\end{tabular}

Tabla V. Principales revistas en las que publica QIOB

El departamento de Química Inorgánica, Orgánica y Bioquímica ha publicado un total de 213 trabajos en 78 revistas. El $51 \%$ de todos los 
trabajos (109) se han publicado en 9 publicaciones científicas, todas ellas con una alta visibilidad por encontrarse en el primer o segundo cuartil de su categoría. La favorita para difundir sus investigaciones es el Journal of Organometallic Chemistry con 27 publicaciones, que representan el $12,7 \%$ de sus trabajos, seguido de European Journal of Inorganic Chemistry. Entre las dos han difundido el $22 \%$ de los estudios.

El departamento de Química Analítica y Tecnología de los Alimentos necesita 7 títulos para publicar el $51,5 \%$ de sus trabajos (89), y un total de 51 para difundir su totalidad, por lo que se puede decir que estas revistas constituyen el núcleo principal. La mayoría están adscritas al primer cuartil de su categoría, y 2 pertenecen al segundo y tercer cuartil. De todas ellas, destaca Analytica Chimica Acta, perteneciente al primer cuartel, en la que se publican 28 trabajos, el $16,2 \%$ del total que producen.

\begin{tabular}{lllcc}
\hline \multicolumn{1}{c}{ Titulo de la revista } & $N^{\circ}$ & $\Sigma$ & $\%$ & $Q$ \\
\hline Analytica Chimica Acta & 28 & 28 & 16,18 & 1 \\
Chromatographia & 12 & 40 & 23,12 & 3 \\
J.Agricultural and Food Chemistry & 12 & 52 & 30,06 & 1 \\
Talanta & 12 & 64 & 36,99 & 1 \\
Food Chemistry & 9 & 73 & 42,20 & 1 \\
Eur. Food Research \& Technology & 8 & 81 & 46,82 & 2 \\
Journal of Chromatography a & 8 & 89 & 51,45 & 1 \\
\hline
\end{tabular}

Tabla VI. Principales revistas en las que publica QATA

\begin{tabular}{lcccc}
\hline \multicolumn{1}{c}{ Titulo de la revista } & $N^{\circ}$ & $\Sigma$ & $\%$ & $Q$ \\
\hline Chemical Physics Letters & 17 & 17 & 11,04 & 2 \\
J.Physical Chemistry a & 12 & 29 & 18,83 & 1 \\
Atmospheric Environment & 7 & 36 & 23,38 & 1 \\
J.Molecular Structure & 6 & 42 & 27,27 & 3 \\
J.Atmospheric Chemistry & 5 & 47 & 30,52 & 2 \\
Spectrochimic Acta Part a- & & & & \\
Molecular \& Biomolecular & 5 & 52 & 33,77 & 3 \\
Biosystems & 4 & 56 & 36,36 & 3 \\
Clay Minerals & 4 & 60 & 38,96 & 2 \\
Int. J.Biochemistry \& Cell Bio & 4 & 64 & 41,56 & 2 \\
Int. J.Chemical Kinetics & 4 & 68 & 44,16 & 3 \\
J. Photochemistry \& Photobiology & 4 & 72 & 46,75 & 3 \\
Biological Chemistry & 3 & 75 & 48,70 & 2 \\
J. Mathematical Chemistry & 3 & 78 & 50,65 & 2 \\
\hline
\end{tabular}

Tabla VII. Principales revistas en las que publica $Q F$

Química Física publicó los 154 trabajos que realiza en 75 revistas diferentes, el $50 \%$ en 13 , y sólo con 3 el 23,5\%: Chemical Physics Letters (17 docs.), Journal of Physical Chemistry (12 docs.) y en Atmospheric Environment (7 docs.).
Estas revistas son de gran visibilidad pues pertenecen al segundo y primer cuartil de su categoría (tabla VII).

Ingeniería Química publica sus 133 trabajos en 47 revistas diferentes. Para difundir el $50 \%$ sólo necesita 8 títulos y con sólo 2 de ellas ven la luz el $30 \%$ de las investigaciones, convirtiéndose en el núcleo de las utilizadas por este departamento. Son: Industrial \& Engineering Chemistry Research (27), y Applied Catálisis a-General (13), ambas con un alta visibilidad internacional por pertenecer al primer y segundo cuartil de su categoría.

\begin{tabular}{lllcc}
\hline \multicolumn{1}{c}{ Título de la revista } & $N^{\circ}$ & $\Sigma$ & $\%$ & $Q$ \\
\hline Ind \& Engineering Chem Researc & 27 & 27 & 20,30 & 1 \\
Applied Catalysis a-General & 13 & 40 & 30,08 & 2 \\
J. Chemical Tech.\& Biotechnology & 6 & 46 & 34,59 & 3 \\
J.Applied Electrochemistry & 5 & 51 & 38,35 & 3 \\
J.Chemical and Engineering Data & 5 & 56 & 42,11 & 2 \\
Clays and Clay Minerals & 4 & 60 & 45,11 & 2 \\
Chemical Engineering Science & 4 & 64 & 48,12 & 1 \\
Chemosphere & 4 & 68 & 51,13 & 1 \\
\hline
\end{tabular}

Tabla VIII. Principales revistas en las que publica IQ

\section{Conclusiones}

Desde la creación de la universidad en el año 1985 se han ido incrementando los fondos destinados a investigación paulatinamente. Si bien en un principio eran únicamente fondos propios, posteriormente comenzó a recibir capital de la Junta de Comunidades y se accedió a los fondos de la Comisión Interministerial de Ciencia y Tecnología (Sánchez, 2003). A partir de ahí se observa una incidencia directa en el incremento de publicaciones científicas que comunican el resultado de la actividad científica y especialmente se aprecia en la productividad de los departamentos del área de la química como principales beneficiarios de estas inversiones. El departamento de Química Inorgánica, Orgánica y Bioquímica es el más productivo de toda la universidad. Sin embargo, hay que destacar el incremento de las publicaciones de Química Analítica y Tecnología de los Alimentos. Hay que tener en cuenta que, según Serrano (2007), la UCLM es la vigésimo tercera universidad española con mayor repercusión investigadora y que atendiendo a los valores del Ranking lberoamericano de Instituciones de Investigación (Ri3) elaborado por el Grupo SClmago, en el área de la química, es la vigésimo cuarta institución más productiva, y durante el período objeto de estudio según su Journal \& Country rank del Grupo produjo, el $3,16 \%$ de la producción na- 
cional en química inorgánica, el $2,4 \%$ en química analítica y el $1,6 \%$ de química orgánica.

En un sistema en el que se tiende con más frecuencia a la interdisciplinaridad en la investigación (Laudel y Origgi, 2006), con políticas de un sistema público de I+D que favorecen la movilidad de los investigadores con el objetivo de adquirir conocimiento en centros internacionales de excelencia (Granadino et al., 2005), no resulta extraño que vaya aumentando la colaboración tanto a nivel nacional como internacional. Los patrones de colaboración son diferentes para cada área científica y, en el caso concreto de la química en la UCLM, el número medio de autores que firmaron un trabajo durante el período objeto de estudio fue de 4,86 y la tasa de documentos coautorados alcanzo el 98,42 lo que nos indica que se trabaja en colaboración con otros autores y no de forma individual, lo que indica un alto porcentaje de colaboración científica.

Existe una colaboración interdepartamental, sobre todo del departamento de Química Inorgánica, Orgánica y Bioquímica, que lo hace con varios departamentos y centros de investigación de la universidad, pero resulta baja entre los cuatro departamentos. A nivel nacional estos departamentos colaboran fundamentalmente con universidades (Complutense y Autónoma) y centros del CSIC de la Comunidad de Madrid, es así tanto por la proximidad geográfica como por encontrarse estas dos universidades entre las más productivas en esta área (Serrano, 2007). La colaboración con Madrid obtiene valores elevados de visibilidad, siendo alto el número de colaboraciones realizadas, lo que hace pensar que se trata de grupos no esporádicos, sino asentados (Olmeda et al., 2006). A nivel internacional, se colabora con instituciones fundamentalmente europeas, del Reino Unido, Francia e Italia. Puede ser el resultado del programa de Acciones Integradas del Ministerio de Ciencia y Tecnología, que financió la movilidad de investigadores y la cooperación con centros de prestigio en esta materia (Granadino et al., 2005).

Todos los departamentos publican en revistas de alta visibilidad internacional adscritas en su mayoría al primer o segundo cuartil de su categoría.

\section{Referencias}

Arencibia Jorge, R.; Araújo Ruíz, J.A.; Collymore Rodríguez, A.; Leyva Rodríguez, Y. (2001). Estudio bibliométrico de la producción científica de la Revista Cenic Ciencias Químicas 1996-2005. // Revista Cenic Ciencias Químicas. 38:1 (2007) 262-267.
Arroyo Alonso, A.; Pulgarín, A.; Gil Leiva, I. (2005). Estudio cienciométrico de la colaboración científica en la Universidad Politécnica de Valencia, España. // Information Research. 11:1. http://informationrs.net/ir/11-1/paper245. html (2006-30-11).

Gómez, I.; Fernández, M. T,; Bordons, M.; Morillo, F. (2003). Región de Murcia: análisis de la producción científica, estudio bibliométrico del período 1998-2000. Murcia: Fundación Séneca, 2003.

Granadino, B.; Plaza, L. M. Vidal C. (2005). Analysis of Spanish scientific output following the Joint Action Program (Acciones Integradas) of the Ministry of Science and Technology (MCYT). // Research Evaluation. 14:2 (2005) 97-102.

Grupo Scimago (2007). La productividad ISI de las universidades españolas (2000-2004). // El profesional de la información. 16:4 (2007) 354-358.

Grupo Scimago (2007). SJR: Scimago Journal \& Country Rank. http:// www.scimagojr/com (2008-05-22).

Laudel, G.; Origgi, G. (2006). Introduction to a special issue on the assessment of interdisciplinary research. // Research evaluation. 15:1 (2006) 2-4.

Martín, N.; Nebot, I. (2004). La calidad de la investigación química. // El País. 9:29 (2004) 39.

Olmeda Gómez, C.; Perianne Rodríguez, A.; Ovalle Perandones, M.A.; Ortiz Repiso, V.; Aragón González, I. (2006). Representaciones de la colaboración autonómica de la Comunidad de Madrid mediante patrones de coautoría (1995-2003). // Guerrero Bote, V.P. (ed.). Proceddings I internacional conference on multidisciplinary information science. Badajoz: Instituto Abierto del Conocimiento, 2006. 625-629.

Prat, A.M. (2001). Evaluación de la producción científica como instrumento para el desarrollo de la ciencia y la tecnología. // Acimed. 9 (2001) 111-114.

Sánchez, I. (2003). Sin universidad no hay desarrollo: XXV años de historia social y económica en Albacete 19772002. Albacete, Instituto de Estudios Albacetenses, 2003.

Sancho, R. (1990). Indicadores bibliométricos en la evaluación de la ciencia y la tecnología: revisión bibliográfica. // Revista Española de Documentación Científica. 13:3-4 (1990) 842-864.

Serrano, P. (2007). La investigación universitaria habla catalán. // La gaceta de los negocios. 25 (Febrero 26). 